\title{
The electron-propagator approach to conceptual density-functional theory
}

\author{
JUNIA MELIN, ${ }^{1}$ PAUL W AYERS ${ }^{2}$ and J V ORTIZ ${ }^{1}$ \\ ${ }^{1}$ Department of Chemistry, Kansas State University, Manhattan, KS 66506-3701, USA \\ ${ }^{2}$ Department of Chemistry, McMaster University, Hamilton, Ontario, Canada L8S 4M1 \\ e-mail: ayers@mcmaster.ca
}

\begin{abstract}
Both electron propagator theory and density-functional theory provide conceptually useful information about chemical reactivity and, most especially, charge transfer. This paper elucidates the qualitative and quantitative links between the two theories, with emphasis on how the reactivity indicators of conceptual density-functional theory can be derived from electron propagator theory. Electron propagator theory could be used to compute reactivity indices with high accuracy at reasonable computational cost.
\end{abstract}

Keywords. Electron-propagator approach; conceptual density-functional theory.

\section{Motivation}

Though there are many approaches to the electronic structure problem, only a few combine quantitative accuracy with qualitative utility. By this standard, density-functional theory (DFT) and electron propagator theory (EPT) stand out. Density-functional theory is exact (in principle) and for at least the past twenty-five years has been used to describe observed chemical trends. ${ }^{1-6}$ However, in the most common implementations of density-functional theory (that is, Kohn-Sham DFT with an approximate exchange-correlation energy functional), ${ }^{7}$ the effects of electron correlation are (sometimes very) imperfectly included. By contrast, electron propagator theory aims at exact solutions to the many-electron Schrödinger equation, so that the accuracy of this approach is more closely attuned to that of conventional, wave-function based, ab initio approaches to quantum chemistry such as many-body perturbation theory and coupled-cluster methods. ${ }^{8-11}$ Electron propagator theory has proved to be very useful for describing the electronic structure of molecules, but it is rarely used to address molecules' reactivity.

The purpose of this paper is to present the links, both qualitative and quantitative, between the theory of the electron propagator and density-functional theory. In particular, we wish to derive an explicit link between the "wave function perspective" of

\footnotetext{
*For correspondence
}

EPT and the "electron density perspective" of DFT. For example, electron propagator theory discloses that the overlap between the many-electron, initial and final state, wave-functions that pertain to the lowest ionization energy is related to the first Dyson orbital, $\phi_{0}^{I P}(\boldsymbol{r})$. Density-functional theory indicates that the change in the electron density associated with ionization is equal to the Fukui function, $f^{-}(\boldsymbol{r}) .^{12,13}$ Since both $\phi_{0}^{I P}(\boldsymbol{r})$ and $f^{-}(\boldsymbol{r})$ are fundamentally related to the process of ionization, they must be related, so we may hypothesize that

$$
f^{-}(\boldsymbol{r})=\left|\phi_{0}^{I P}(\boldsymbol{r})\right|^{2}+(\text { other terms }) \text {. }
$$

One of the primary goals of this paper is to derive the form of the "other terms" in (1). More generally, we wish to derive expressions for the global and local reactivity indices in conceptual DFT in terms of quantities related to electron propagator theory, so that we can more fully answer the fundamental question: "How are the electron propagator and density-functional perspectives related?".

Quite aside from the latter question's fundamental importance, our goal is to demonstrate how global and local reactivity indicators in density-functional theory can be evaluated using electron-propagator theory. The reactivity indicators of conceptual DFT, however, are completely defined within the exact DFT, and are totally independent of the Kohn-Sham ansatz and any approximation thereto. Consequently, it is entirely appropriate to compute reactivity indi- 
cators using any available method, and the equations we derive make it feasible to use ab initio techniques to compute accurate approximations to reactivity indicators. Not only is this appropriate, but using $a b$ initio techniques may be highly desirable, especially since, as commonly applied, Kohn-Sham density-functional theory sometimes struggles to compute quantities related to reaction mechanisms (e.g., barrier heights). ${ }^{14,15}$ Electron propagator techniques may provide a way forward, allowing reactivity indicators to be computed more reliably.

To these ends, $\S \S 2$ and 3 of this paper present the requisite background information on conceptual density-functional theory and electron propagator theory. Next, we consider how electron propagator theory can be used to compute global and local reactivity indicators ( $\S \S 4$ and 5 respectively). Section 6 concludes.

\section{Conceptual density-functional theory}

Readers familiar with conceptual density-functional theory ${ }^{16,17}$ may wish to skip to the next section, as the following discussion and notation is standard. The intent is to present the key quantities in conceptual density-functional theory to a more general audience.

The key insight underlying density-functional theory is that the electronic energy of a molecule can be considered to be a functional of the electron density, ${ }^{18}$

$$
\rho(x)=\left\langle\Psi\left|\sum_{i=1}^{N} \delta\left(r_{i}-x\right)\right| \Psi\right\rangle
$$

or, equivalently, of the external potential, $v(\boldsymbol{x}) \cdot{ }^{19}$ The external potential is just the potential felt by electrons in a molecule due to entities that are not electrons. In the simplest case, the external potential is just the potential due to the atomic nuclei,

$$
v(\boldsymbol{x})=\sum_{\alpha=1}^{P} \frac{-Z_{\alpha}}{\left|\boldsymbol{x}-\boldsymbol{R}_{\alpha}\right|},
$$

where $\left\{\boldsymbol{R}_{\alpha}\right\}_{\alpha=1}^{P}$ and $\left\{Z_{\alpha}\right\}_{\alpha=1}^{P}$ denote the positions and atomic numbers of the atomic nuclei in a $P$-atom molecule. More generally, the external potential includes the potential due to external fields, solvation, and in quantum mechanics/molecular mechanics (QM/MM) hybrid calculations, other molecules (or other pieces of the same macromolecule).
Following Hohenberg and Kohn, ${ }^{18}$ the electronic energy can be written as a functional of the electron density

$$
E_{v}[\rho]=T[\rho]+V_{e e}[\rho]+\int \rho(\boldsymbol{x}) v(\boldsymbol{x}) \mathrm{d} \boldsymbol{x}
$$

where $T[\rho]$ and $V_{e e}[\rho]$ denote the kinetic energy and electron-electron repulsion energy functionals. In (4), the external potential is fixed by identity of the molecular system and is thus treated as a parameter and not a variable. Equation (4) is an exact expression for the energy of a molecule, complicated only by the fact that computationally useful forms for $T[\rho]$ and $V_{e e}[\rho]$ are unknown. (Exact, but computationally impracticable, expressions are available, however. ${ }^{20-23}$ ) Kohn and Sham partially circumvented these problems by rewriting (4) in the form, ${ }^{7}$

$$
E_{v}[\rho]=T_{s}[\rho]+J[\rho]+E_{x c}[\rho]+\int \rho(\boldsymbol{x}) v(\boldsymbol{x}) \mathrm{d} \boldsymbol{x},
$$

where $T_{s}[\rho]$ is the kinetic energy of the reference system of non-interacting electrons that has the same density as the interacting system,

$$
J[\rho]=\frac{1}{2} \iint \frac{\rho(x) \rho\left(x^{\prime}\right)}{\left|x-x^{\prime}\right|} \mathrm{d} x \mathrm{~d} \boldsymbol{x}^{\prime},
$$

is the classical coulomb repulsion between electrons, and $E_{x c}[\rho]$ is the exchange-correlation energy. Both $T_{s}[\rho]$ and $J[\rho]$ can be exactly computed; the exact form of $E_{x c}[\rho]$ is not known but good approximations exist. Significantly, because the exchangecorrelation energy is a rather small fraction of the total electronic energy, relatively large relative errors in the exchange-correlation energy functional need not cause unacceptably large absolute errors in the total energy. (This is not true for the unknown functionals in (4), which are similar in magnitude to the total energy.)

The historical roots of conceptual - as opposed to computational - DFT can be traced to a 1978 paper by Parr, Donnelly, Levy, and Palke. ${ }^{24}$ There they observed that the fundamental variational principle in DFT could be written in the form

$$
\frac{\delta E_{v}[\rho]}{\delta \rho(\boldsymbol{r})}=\mu
$$

where $\mu$, which they called the electronic chemical potential, is 


$$
\mu=\left(\frac{\partial E}{\partial N}\right)_{v(r)}
$$

Following Itzowski and Margrave, ${ }^{25}$ and observing that a finite-difference approximation to the chemical potential was just the additive inverse of the Mulliken electronegativity, ${ }^{26}$

$$
\mu \approx-(I+A) / 2=-\chi_{\text {Mulliken }},
$$

Parr et al concluded that the electronegativity should be defined as minus the electronic chemical potential.

From this base, a plethora of new reactivity indicators were first proposed and later systematized using analogies to thermodynamics, perturbation theory, and variational principles. ${ }^{1,3,6,17,27-31}$ In each case, a key mathematical concept is the functional derivative, to which we have already been introduced in (7). Just as the derivative of a function indicates how the value of the function changes when the argument changes

$$
\begin{aligned}
& f(x+\mathrm{d} x)-f(x) \approx(\mathrm{d} f / \mathrm{d} x) \mathrm{d} x \\
& f(\boldsymbol{x}+\mathrm{d} \boldsymbol{x})-f(\boldsymbol{x}) \approx \nabla f(\boldsymbol{x}) \cdot \mathrm{d} \boldsymbol{x},
\end{aligned}
$$

the functional derivative encapsulates information about how a functional changes when its argument changes,

$$
E_{v}[\rho(\boldsymbol{x})+\delta \rho(\boldsymbol{x})]-E_{v}[\rho[\boldsymbol{x}]] \approx \int \frac{\delta E[\rho]}{\delta \rho(\boldsymbol{x})} \delta \rho(\boldsymbol{x}) \mathrm{d} \boldsymbol{x} .
$$

The integral in (12) is required because the function (in this case, the electron density) may change in many places at once, and so we must take the Riemann sum (i.e. integral) over all the possible locations where the density might change. If we restrict ourselves to the special case where the electron density changes only at one point, i.e.

$$
\delta \rho(x)=\varepsilon \delta\left(x-x_{0}\right),
$$

then $E_{v}\left[\rho(\boldsymbol{x})+\varepsilon \delta\left(\boldsymbol{x}-\boldsymbol{x}_{0}\right)\right]-E_{v}[\rho[\boldsymbol{x}]]$

$$
\begin{gathered}
\approx \varepsilon \int[\delta E[\rho] / \delta \rho(\boldsymbol{x})] \delta\left(\boldsymbol{x}-\boldsymbol{x}_{0}\right) \mathrm{d} \boldsymbol{x} \\
\frac{E_{v}\left[\rho(\boldsymbol{x})+\varepsilon \delta\left(\boldsymbol{x}-\boldsymbol{x}_{0}\right)\right]-E_{v}[\rho[\boldsymbol{x}]]}{\varepsilon} \approx \frac{\delta E[\rho]}{\delta \rho\left(\boldsymbol{x}_{0}\right)} .
\end{gathered}
$$

In the limit of infinitesimal $\varepsilon$, terms proportional to $\varepsilon^{2}$ and higher powers of $\varepsilon$ become negligible, so

$$
\begin{aligned}
& \frac{\delta E[\rho]}{\delta \rho\left(x_{0}\right)} \equiv \underbrace{\lim _{\varepsilon \rightarrow 0}}_{\varepsilon \rightarrow 0} \frac{E_{v}\left[\rho(\boldsymbol{x})+\varepsilon \delta\left(\boldsymbol{x}-\boldsymbol{x}_{0}\right)\right]-E_{v}[\rho[\boldsymbol{x}]]}{\varepsilon} \\
& =\left(\frac{\partial E\left[\rho(\boldsymbol{x})+\varepsilon \delta\left(\boldsymbol{x}-\boldsymbol{x}_{0}\right)\right]}{\partial \varepsilon}\right)_{\varepsilon=0} .
\end{aligned}
$$

The functional derivative can be interpreted as the change in the value of the functional (here, the energy) when the value of the function (here, the electron density) is changed at the point $\boldsymbol{x}_{0}$. The functional derivative can be determined using either (15) or the more fundamental and mathematically rigorous (12). For a demonstration of how (7) is derived from the variational principle for the energy and (15), the reader is referred to [ref. 1].

Based on the insight that chemical reactions are typified by favorable (that is, energy lowering) interaction between reactant molecules, densityfunctional reactivity indices are related to how the energy of a molecule changes as the electron density, external potential, or number of electrons changes due to a perturbation representing the other reagent. The most commonly used reactivity indicators are related to how the electronic energy changes as the number of electrons and external potential changes, thus:

$$
\begin{aligned}
& E[v(\boldsymbol{r})+\Delta v(\boldsymbol{r}) ; N+\Delta N]=E[v(\boldsymbol{r}) ; N] \\
& +\Delta N .\left(\frac{\partial E[v ; N]}{\partial N}\right)_{v(\boldsymbol{r}), N}+\frac{(\Delta N)^{2}}{2} \cdot\left(\frac{\partial^{2} E[v ; N]}{\partial N^{2}}\right)_{v(\boldsymbol{r}), N}+\ldots \\
& \quad+\int\left(\frac{\delta E[v ; N]}{\delta v(\boldsymbol{r})}\right)_{v(\boldsymbol{r}), N} \Delta v(\boldsymbol{r}) \mathrm{d} \boldsymbol{r} \\
& \quad+\frac{1}{2} \iint \Delta v\left(\boldsymbol{r}^{\prime}\right)\left(\frac{\delta^{2} E[v ; N]}{\delta v(\boldsymbol{r}) \delta v\left(\boldsymbol{r}^{\prime}\right)}\right)_{N} \Delta v(\boldsymbol{r}) \mathrm{d} \boldsymbol{r} \mathrm{d} \boldsymbol{r}^{\prime}+\ldots \\
& \quad+\Delta N \int\left(\frac{\delta \partial E[v ; N]}{\delta v(\boldsymbol{r}) \partial N}\right)_{v(\boldsymbol{r}), N} \Delta v(\boldsymbol{r}) \mathrm{d} \boldsymbol{r}+\cdots
\end{aligned}
$$

Note that all the derivatives and functional derivatives are evaluated for the unperturbed system. Thus, conceptual density-functional theory strives to explain the molecular reactivity using only properties of the isolated reactants. 
It should also be noted that (16) could be written down without any recourse to density-functional theory. The association of (16) to density-functional theory is mostly historical: the language, literature, and methods typically used to interpret equations of this form are associated with density-functional theory. There is no compelling reason, though, not to use an alternative method (e.g., electron propagator theory) to evaluate the quantities appearing in (16).

In fact, the terms in (16) are often discussed using vocabulary more often associated with molecularorbital theory. Using Klopman's classification of different chemical reaction types, ${ }^{32}$ the terms on the second line, which depend on the change in the number of electrons but not the change in external potential, are typically important in "frontier orbital controlled" chemical reactions. The first term, here, can be identified with the electronic chemical potential (compare (8)) while the second term is associated with the chemical hardness, ${ }^{33}$

$$
\eta=\left(\frac{\partial^{2} E}{\partial N^{2}}\right)_{v(r)} \approx I-A .
$$

The relationship between $\eta$ and the ionization potential and electron affinity of the molecule was first proposed by Parr and Pearson and is based on the assumption that the electronic energy is a quadratic function of the number of electrons. ${ }^{33}$ The equalization of the electronic chemical potential follows by analogy to classical thermodynamics, yielding the electronegativity equalization principle. ${ }^{24,34}$ The chemical hardness is important for explaining the maximum hardness ${ }^{29,35-38}$ and hard/soft acid/base principles. ${ }^{39-41}$ When information about the chemical hardness is combined with information from the chemical potential, reactivity indices related to the power of electrophiles, ${ }^{42}$ nucleophiles, ${ }^{43}$ and leaving groups $^{44,45}$ can be derived.

The next two terms, which depend on the change in external potential but not the amount of electron transfer, are associated with "charge controlled" reactions in the Klopman classification. ${ }^{32}$ The first of these two terms is related to the electrostatic potential,$~^{46}$

$$
\Phi(\boldsymbol{r})=\sum_{\alpha=1}^{P} \frac{Z_{\alpha}}{\left|\boldsymbol{x}-\boldsymbol{R}_{\alpha}\right|}-\int \frac{\rho\left(\boldsymbol{x}^{\prime}\right)}{\left|\boldsymbol{x}-\boldsymbol{x}^{\prime}\right|} \mathrm{d} \boldsymbol{x}^{\prime},
$$

while the second term is related to the polarization of the reactant by attacking reagent, and is often ne- glected. Deriving the relationship between (16) and the electrostatic potential requires one to know that

$$
\left(\frac{\delta E[v ; N]}{\delta v(\boldsymbol{x})}\right)_{v(\boldsymbol{r}), N}=\rho(\boldsymbol{x}),
$$

which follows directly from (15), the HellmannFeynman theorem, and the definition of the electron density. If we consider the change in external potential to be due to an infinitesimal positive point charge, then inserting (19) into (16) and adding the term due to nuclear repulsion yields the electrostatic potential, (18). The fourth and fifth terms are the basis for "external potential-based" reactivity indices. ${ }^{30}$

The last line in (16) represents the coupling between changes in external potential and changes in electron number. Using either (8) or (19), we can simplify the functional derivative to

$$
f(\boldsymbol{r})=\left(\frac{\partial \rho(\boldsymbol{r})}{\partial N}\right)_{v(\boldsymbol{r})}=\left(\frac{\delta \mu}{\delta v(\boldsymbol{r})}\right)_{N} .
$$

$f(\boldsymbol{r})$ is called the Fukui function; ${ }^{13,47}$ it is related to the frontier orbitals but it includes the effects of electron correlation and orbital relaxation. ${ }^{12}$ Since the Fukui function does not make an important contribution to the expression for the molecular interaction energy (cf. (16)) unless there is significant charge transfer between the reactants, it is usually important for "frontier orbital controlled" reactions. When orbital relaxation and/or electron correlation are important, the Fukui function is a more accurate predictor of regioselectivity than the frontier molecular orbitals. ${ }^{48-50}$

An important and subtle issue arises when considering formulae like (8), where the energy is differentiated with respect to the number of electrons. The number of electrons is always an integer, so it is by no means assured that we can treat the number of electrons as a continuous variable. In the usual approach, we consider the number of electrons to be defined by introducing a heat bath, and then taking the zero temperature limit. ${ }^{51}$ An alternative is to derive the result based on the constraint that the underlying exact functional must be size consistent. ${ }^{52}$ (Though this is not strictly required, it is a useful additional constraint to impose.) Either approach yields the same final result, which is that the energy, electron density, and any other size-consistent functional of fractional-electron systems is simply given 
by the linear interpolation between the systems with integer electron number. Owing to this, it is necessary to define derivatives with respect to the number of electrons from above and below, giving ${ }^{13,29,47,51}$

$$
\begin{aligned}
& \mu^{+}=\left(\frac{\partial E}{\partial N}\right)_{v(\boldsymbol{r})}^{+}=-A \\
& \mu^{-}=\left(\frac{\partial E}{\partial N}\right)_{v(\boldsymbol{r})}^{-}=-I \\
& f^{+}(\boldsymbol{r})=\left(\frac{\delta \mu^{+}}{\delta v(\boldsymbol{r})}\right)_{N}=\left(\frac{\partial \rho(\boldsymbol{r})}{\partial N}\right)_{v(\boldsymbol{r})}^{+}=\rho_{N+1}(\boldsymbol{r})-\rho_{N}(\boldsymbol{r}) \\
& f^{-}(\boldsymbol{r})=\left(\frac{\delta \mu^{-}}{\delta v(\boldsymbol{r})}\right)_{N}=\left(\frac{\partial \rho(\boldsymbol{r})}{\partial N}\right)_{v(\boldsymbol{r})}^{-}=\rho_{N}(\boldsymbol{r})-\rho_{N-1}(\boldsymbol{r}) .
\end{aligned}
$$

Equations (21) and (22) are exact for exact calculations, but are usually only approximate. In (22), we have chosen to explicitly denote the number of electrons in the reactant molecule with a subscript on the electron density. Clearly the Fukui function measures how the electron density changes when we remove an electron from the system $\left(f^{-}(\boldsymbol{r})\right)$ or add an electron to the system $\left(f^{+}(\boldsymbol{r})\right)$. Since electrophilic attack tends to occur in locations where molecules are willing to donate electrons (where $f^{-}(\boldsymbol{r})$ is large) and nucleophilic attack tends to occur in locations where molecules are eager to accept electrons (where $f^{+}(\boldsymbol{r})$ is large), it is not surprising that the Fukui functions encapsulate information about regioselectivity. Thus, though it is mathematical inconvenient, the derivative discontinuity with respect to electron number is chemically important, since it predicts that ambiphilic molecules respond differently to nucleophilic and electrophilic attack.

The biggest drawback of the derivative discontinuity is that the chemical hardness becomes ill-defined (it is zero for one-sided derivatives and infinite for the two-sided derivative). However, some information remains since we can write ${ }^{29}$

$$
\begin{aligned}
& \eta(M)=\left(\frac{\partial^{2} E}{\partial N^{2}}\right)_{v(r)}=(I-A) \delta(M-N), \\
& N-1<M<N+1 .
\end{aligned}
$$

Here again $N$ is the number of electrons in the isolated reactant molecule.

\section{Electron propagator theory}

The Fukui function is a key quantity in densityfunctional theory because it expresses how the electron density - the fundamental descriptor of chemical systems in DFT - changes as the number of electrons changes. Dyson orbitals play a similar role in electron propagator theory: Dyson orbitals express how the wave function - the fundamental descriptor in conventional $a b$ initio approaches to the electronic structure problem - changes as the number of electrons changes. Just as we have Fukui functions for electron attachment and electron removal, we have Dyson orbitals for electron attachment,

$$
\begin{aligned}
\phi_{l}^{E A}(z) \equiv \sqrt{N+1}[ & \cdots \int\left(\Psi_{0}^{(N)}\left(z_{1}, \ldots, z_{N}\right)\right)^{*} \\
& \left.\times \Psi_{l}^{(N+1)}\left(z_{1}, \ldots, z_{N}, z\right) \mathrm{d} z_{1} \ldots \mathrm{d} z_{N}\right],
\end{aligned}
$$

and electron removal,

$$
\begin{aligned}
\phi_{k}^{I P}(z) \equiv \sqrt{N}\left[\int\right. & \ldots \int\left(\Psi_{k}^{(N-1)}\left(z_{1}, \ldots, z_{N-1}\right)\right)^{*} \\
& \left.\times \Psi_{0}^{(N)}\left(z_{1}, \ldots, z_{N-1}, z\right) \mathrm{d} z_{1} \ldots \mathrm{d} z_{N-1}\right] .
\end{aligned}
$$

Here $\Psi_{m}^{(M)}\left(z_{1}, \ldots, z_{M}\right)$ denotes the $m$ th eigenfunction of the $M$-electron system and $z_{i}$ denotes both the spatial and spin coordinates of the $i$ th electron. The factors of $\sqrt{N+1}$ and $\sqrt{N}$ in (24) and (25), respectively, arise from normalization of the antisymmetric, many-electron wavefunctions.

Based on their definitions, (24) and (25), one might suspect that the Dyson orbitals require that all the eigenstates of the $N+1$ and $N-1$ electron systems be determined, in addition to the usual determination of the $\mathrm{N}$-electron ground state. However, within the purview of electron propagator theory, the Dyson orbitals and the associated ionization potentials and electron affinities can be determined from a single calculation on the $N$-electron system. To see how this arises, recall that for a one-electron Hamiltonian, $\hat{f}(z)$, the electron propagator, $G\left(z, z^{\prime}, \varepsilon\right)$ solves the equation

$$
(\varepsilon-\hat{f}(z)) G\left(z, z^{\prime}, \varepsilon\right)=\delta\left(z-z^{\prime}\right)
$$

whence 


$$
\begin{aligned}
G\left(z, z^{\prime}, \varepsilon\right) & =(\varepsilon-\hat{f}(z))^{-1} \\
& =\sum_{k=0}^{\infty} \frac{\psi_{k}(z) \psi_{k}^{*}\left(z^{\prime}\right)}{\varepsilon-\varepsilon_{k}} .
\end{aligned}
$$

In the first line of $(27),(\varepsilon-\hat{f}(z))^{-1}$ represents the inverse of an operator, and not merely a multiplicative inverse. In the second line of $(27),\left\{\psi_{k}(z)\right\}$ and $\left\{\varepsilon_{k}\right\}$ are the eigenfunctions and eigenvalues of the 1electron Hamiltonian,

$$
\hat{f}(z) \psi_{k}(z)=\varepsilon_{k} \psi_{k}(z) .
$$

$\hat{f}(z)$ can be any one-electron Hamiltonian, and while there have been many studies using the KohnSham Hamiltonian in this context, ${ }^{53-61}$ our primary goal is to draw the link between conceptual DFT and conventional $a b$ initio approaches, so we shall henceforth consider $\hat{f}(z)$ to be the Hartree-Fock operator.

If we consider a system of $N$ non-interacting electrons defined by the Hamiltonian

$$
\hat{H}^{(H F)}=\sum_{i=1}^{N} \hat{f}\left(z_{i}\right)
$$

the ground state wave function will be a Slater determinant composed of the Hartree-Fock spin-orbitals

$$
\Psi_{0}^{(N, H F)}=\left|\psi_{0} \psi_{1} \ldots \psi_{N-1}\right|
$$

The occupied Hartree-Fock orbitals are, according to Koopmans's theorem, associated with ionization

$$
\begin{aligned}
& I_{k}^{(H F)}=-\varepsilon_{k} \quad 0 \leq k \leq N-1 \\
& \phi_{k}^{(I P, H F)}\left(z_{N}\right) \\
& =\left.\sqrt{N}\left\langle\left|\psi_{0} \ldots \psi_{k-1} \psi_{k+1} \ldots \psi_{N-1}\right| \mid \psi_{0} \ldots \psi_{N-1}\right\rangle\right|_{1 \ldots N-1}
\end{aligned}
$$

where the notation in the second line indicates integration with respect to the coordinates of the first $N-1$ electronic coordinates. If we adopt the convention that $\phi_{k}^{(I P, H F)}\left(z_{N}\right)=0$ for $k \geq N$, then

$$
\phi_{k}^{(I P, H F)}(z)= \begin{cases}\Psi_{k}(z), & N>k \\ 0, & N \leq k\end{cases}
$$

(This convention is most natural when second quantization is used, but we avoid using second quantization in this paper because some in the DFT community are unfamiliar with this notation. Second quantization is not commonly employed in DFT because the electron density (and related quantities) are associated with a nonreflexive Banach space, whereas the algebraic structure of second quantization requires a graded Hilbert space which can be generalized to reflexive Banach spaces.) It is useful to recall the formula for the Hartree-Fock energy in terms of the orbital energies of the occupied orbitals and expectation values of the one-electron Hamiltonian, namely,

$$
E^{(H F)}=\frac{1}{2}\left(\sum_{k=0}^{N-1} \varepsilon_{k}+\sum_{k=0}^{N-1}\left\langle\psi_{k}\left|-\frac{\nabla^{2}}{2}+v(\boldsymbol{r})\right| \psi_{k}\right\rangle\right) .
$$

Similarly, the unoccupied Hartree-Fock orbitals are associated with electron attachment,

$$
\begin{aligned}
& A_{l}^{(H F)}=-\varepsilon_{l} \quad N \leq l \\
& \phi_{l}^{(E A, H F)}\left(z_{N+1}\right) \\
& \quad=\sqrt{N+1}\left\langle\left|\psi_{0} \ldots \psi_{N-1}\right||| \psi_{0} \ldots \psi_{N-1} \psi_{l} \mid\right\rangle_{1 \ldots N} .
\end{aligned}
$$

Because of the Pauli exclusion principle,

$$
\phi_{l}^{(E A, H F)}(z)= \begin{cases}\psi_{l}(z), & N \leq l, \\ 0, & N>l .\end{cases}
$$

Based on (31)-(37), we see that the Hartree-Fock occupied orbitals are analogous to the Dyson orbitals for electron removal, $\left\{\phi_{k}^{I P}\right\}$, and the Hartree-Fock virtual orbitals are analogous to the Dyson orbitals for electron attachment $\left\{\phi_{l}^{E A}\right\}$. Thus, the one-electron propagator in Hartree-Fock theory can be rewritten as

$$
\begin{aligned}
G^{(H F)}\left(z, z^{\prime}, \varepsilon\right)=\sum_{k=0}^{\infty} & \frac{\phi_{k}^{(I P, H F)}(z)\left(\phi_{k}^{(I P, H F)}\left(z^{\prime}\right)\right)^{*}}{\varepsilon-\varepsilon_{k}} \\
& +\sum_{l=0}^{\infty} \frac{\phi_{l}^{(E A, H F)}(z)\left(\phi_{l}^{(E A, H F)}\left(z^{\prime}\right)\right)^{*}}{\varepsilon-\varepsilon_{l}} .
\end{aligned}
$$

which may be chosen to be the zero-order electron propagator, $G_{0}\left(z, z^{\prime}, \varepsilon\right)$. This leads us to propose the following definition for the exact one-electron propagator,

$$
\begin{aligned}
G\left(z, z^{\prime}, \varepsilon\right)= & \sum_{k=0}^{\infty} \frac{\phi_{k}^{I P}(z)\left(\phi_{k}^{I P}\left(z^{\prime}\right)\right)^{*}}{\varepsilon+I_{k}} \\
& +\sum_{l=0}^{\infty} \frac{\phi_{l}^{E A}(z)\left(\phi_{l}^{E A}\left(z^{\prime}\right)\right)^{*}}{\varepsilon+A_{l}} .
\end{aligned}
$$


Approximations to the electron propagator are usually constructed in terms of an energy-dependent potential, the self-energy operator, $\sum(x, \varepsilon)$. The Dyson equation,

$$
\begin{aligned}
G\left(z, z^{\prime}, \varepsilon\right)= & G_{0}\left(z, z^{\prime}, \varepsilon\right)+\int G(z, x, \varepsilon) \\
& \sum(\boldsymbol{x}, \varepsilon) G_{0}\left(\boldsymbol{x}, \boldsymbol{z}^{\prime}, \varepsilon\right) \mathrm{d} \boldsymbol{x} \\
= & G_{0}\left(\boldsymbol{z}, \boldsymbol{z}^{\prime}, \varepsilon\right)+\int G(\boldsymbol{z}, \boldsymbol{x}, \varepsilon) \\
& \quad(\boldsymbol{x}, \varepsilon) \delta\left(\boldsymbol{x}-\boldsymbol{x}^{\prime}\right) G_{0}\left(\boldsymbol{x}^{\prime}, \boldsymbol{z}^{\prime}, \varepsilon\right) \mathrm{d} \mathbf{x} \mathrm{d} \boldsymbol{x}^{\prime},
\end{aligned}
$$

can be solved directly by iterative approximation or by insertion of the left-hand side into the right-hand side, yielding

$$
\begin{aligned}
& G\left(z, z^{\prime}, \varepsilon\right)=G_{0}\left(z, z^{\prime}, \varepsilon\right) \\
& +\int G_{0}\left(z, x_{1}, \varepsilon\right) \Sigma\left(x_{1}, \varepsilon\right) \delta\left(x_{1}-x_{1}^{\prime}\right) G_{0}\left(x_{1}^{\prime}, z^{\prime}, \varepsilon\right) \mathrm{d} x_{1} \mathrm{~d} x_{1}^{\prime} \\
& +\iint G_{0}\left(z, x_{1}, \varepsilon\right) \Sigma\left(x_{1}, \varepsilon\right) \delta\left(x_{1}-x_{1}^{\prime}\right) G_{0}\left(x_{1}^{\prime}, x_{2}, \varepsilon\right) \\
& \quad \times \Sigma\left(x_{2}, \varepsilon\right) \delta\left(x_{2}-x_{2}^{\prime}\right) G_{0}\left(x_{2}^{\prime}, z^{\prime}, \varepsilon\right) \mathrm{d} x_{1} \mathrm{~d} x_{1}^{\prime} \mathrm{d} x_{2} \mathrm{~d} x_{2}^{\prime} \\
& +\ldots
\end{aligned}
$$

It is worth noting that (40) is exact in all circumstances, while equations based on (41) rely on the assumed convergence of the Taylor series. (Based on the questionable convergence of Moller-Plesset Perturbation Theory, this may not always be a justified assumption. ${ }^{62}$ )

Multiplying (40) by the both the operator inverse of the exact and approximate propagators, we obtain

$$
\begin{aligned}
& \iint(G(\boldsymbol{y}, z, \varepsilon))^{-1} G\left(z, z^{\prime}, \varepsilon\right)\left(G_{0}\left(z^{\prime}, y^{\prime}, \varepsilon\right)\right)^{-1} \mathrm{~d} z \mathrm{~d} z^{\prime} \\
& =\iint(G(y, z, \varepsilon))^{-1} G_{0}\left(z, z^{\prime}, \varepsilon\right)\left(G_{0}\left(z^{\prime}, y^{\prime}, \varepsilon\right)\right)^{-1} \mathrm{~d} z \mathrm{~d} z^{\prime} \\
& +\iiint(G(\boldsymbol{y}, z, \varepsilon))^{-1} G(z, x, \varepsilon) \Sigma(x, \varepsilon) \delta\left(x-x^{\prime}\right) \\
& G_{0}\left(x^{\prime}, z^{\prime}, \varepsilon\right)\left(G_{0}\left(z^{\prime}, y^{\prime}, \varepsilon\right)\right)^{-1} \mathrm{~d} z \mathrm{~d} z^{\prime} \mathrm{d} x \mathrm{~d} x^{\prime}, \\
& \iint \delta\left(\boldsymbol{y}-z^{\prime}\right)\left(G_{0}\left(z^{\prime}, y^{\prime}, \varepsilon\right)\right)^{-1} \mathrm{~d} z^{\prime} \\
& =\iint(G(\boldsymbol{y}, \boldsymbol{z}, \varepsilon))^{-1} \delta\left(\boldsymbol{z}-\boldsymbol{y}^{\prime}\right) \mathrm{d} \boldsymbol{z} \\
& +\iiint \delta(\boldsymbol{y}-\boldsymbol{x}) \Sigma(\boldsymbol{x}, \varepsilon) \delta\left(\boldsymbol{x}^{\prime}-\boldsymbol{y}^{\prime}\right) \mathrm{d} \boldsymbol{x} \mathrm{d} \boldsymbol{x}^{\prime} \\
& \iint \delta\left(y-z^{\prime}\right)\left(G_{0}\left(z^{\prime}, y^{\prime}, \varepsilon\right)\right)^{-1} \mathrm{~d} z^{\prime} \\
& =\iint(G(y, z, \varepsilon))^{-1} \delta\left(z-y^{\prime}\right) \mathrm{d} z \\
& +\iiint \delta(y-x) \Sigma\left(x^{\prime}, \varepsilon\right) \delta\left(x-x^{\prime}\right) \delta\left(x^{\prime}-y^{\prime}\right) \mathrm{d} \boldsymbol{x} \mathrm{d} x^{\prime}
\end{aligned}
$$

which gives an explicit expression for the self energy, namely,

$$
\begin{aligned}
\Sigma(\boldsymbol{y}, \varepsilon) \delta\left(\boldsymbol{y}-\boldsymbol{y}^{\prime}\right)= & \left(G_{0}\left(\boldsymbol{y}, \boldsymbol{y}^{\prime}, \varepsilon\right)\right)^{-1} \\
& -\left(G\left(\boldsymbol{y}^{\prime}, \boldsymbol{y}^{\prime}, \varepsilon\right)\right)^{-1}
\end{aligned}
$$

or in matrix notation,

$$
\sum(\varepsilon)=\boldsymbol{G}_{0}^{-1}-\boldsymbol{G}^{-1},
$$

where $\sum(\varepsilon)$ is defined as the projection of $\sum(x, \varepsilon) \delta\left(x-x^{\prime}\right)$ onto a basis set.

In terms of the self-energy, we also have direct expressions for the Dyson orbitals, namely,

$$
\begin{gathered}
(\hat{f}(z)+\hat{\Sigma}(z, \varepsilon)) \phi_{k}^{I P}(z)=-I_{k} \phi_{k}^{I P}(z) \\
(\hat{f}(z)+\hat{\Sigma}(z, \varepsilon)) \phi_{l}^{E A}(z)=-A_{l} \phi_{l}^{E A}(z) .
\end{gathered}
$$

The fundamental goal of electron propagator theory is to find practical approximate expressions for the self-energy operator and to solve the resulting equations. This energy-dependent potential can be expanded in a perturbative series where the lowest correction occurs at second order.

$$
\begin{aligned}
& \sum_{p q}^{(2)}(z, \varepsilon)= \\
& \frac{1}{2}\left[\begin{array}{l}
\sum_{i a b} \frac{\left|\chi_{p}(z)\right\rangle\left\langle\chi_{p} \psi_{i} \| \psi_{a} \psi_{b}\right\rangle\left\langle\psi_{a} \psi_{b} \| \chi_{q} \psi_{i}\right\rangle\left\langle\chi_{q}(z)\right|}{\varepsilon-\varepsilon_{i}-\varepsilon_{a}-\varepsilon_{b}} \\
+\sum_{i j a} \frac{\left|\chi_{q}(z)\right\rangle\left\langle\chi_{q} \psi_{a} \| \psi_{i} \psi_{j}\right\rangle\left\langle\psi_{i} \psi_{j} \| \chi_{p} \psi_{a}\right\rangle\left\langle\chi_{p}(z)\right|}{\varepsilon-\varepsilon_{a}-\varepsilon_{i}-\varepsilon_{j}}
\end{array}\right]
\end{aligned}
$$

here, $i, j$ index the occupied spin-orbitals, $a, b$ index the virtual spin-orbitals, and $\left\{\chi_{p}\right\}$ is a set of orthonormal basis functions. The expression for the thirdorder correction is more complicated. Because the primary purpose of this paper is to derive general relations between electron propagator and DFT, we will defer a treatment of third order contributions to a separate paper.

Once we derive an approximate expression for the self-energy (e.g., (46)) and solve (45) for the Dyson orbitals, we can determine many other quantities, including the $N$-electron charge-density,

$$
\rho_{N}(z)=\sum_{k=0}^{\infty}\left|\phi_{k}^{I P}(z)\right|^{2}
$$


and the pole strengths,

$$
\begin{aligned}
p_{k}^{I P} & =\left\langle\phi_{k}^{I P} \mid \phi_{k}^{I P}\right\rangle \\
& =1+\int\left(\phi_{k}^{I P}(z)\right)^{*}\left(\frac{\partial \Sigma(z,-I)}{\partial I}\right)_{I=I_{k}} \phi_{k}^{I P}(z) \mathrm{d} z,
\end{aligned}
$$

which are related to the intensities of transitions in photoelectron spectra. For better understanding of this quantity it might be useful to recall that, when an electron is removed from a molecule, different processes can take place, such as a simple detachment of the electron from an orbital (as described by the Koopmans's picture of ionization processes), or ionization coupled to excitations, known as shake-ups. Pole strengths can be interpreted as the probability associated to ionization processes given by Koopmans's picture.

In analogy to (34), there is an expression for the electronic energy in terms of the Dyson orbitals, their energies, and their pole strengths:

$$
E_{0}^{(N)}=\frac{1}{2} \sum_{k=0}^{\infty}\left[p_{k}\left(-I_{k}\right)+\left\langle\phi_{k}^{I P}\left|-\frac{\nabla^{2}}{2}+v(\boldsymbol{r})\right| \phi_{k}^{I P}\right\rangle\right] .
$$

Equation (49) clearly reduces to (34) if the manyelectron Hamiltonian is replaced by (29).

\section{Global indicators}

\subsection{Exact theory}

Just as in conceptual DFT, the key quantities needed to describe electron transfer processes are available in electron propagator theory. The eigenvalues of (45) give access to the electron affinities and ionization potentials, while the Dyson orbitals encapsulate information about the changes in a wave-function associated with ionization and electron attachment processes. Consequently, we can derive the reactivity indicators defined in conceptual DFT in terms of electron propagator formalism.

If one uses (21) to model the chemical potential, then the eigenvalues of the Dyson equation fully determine the chemical potential from above and below. It is sometimes useful, however, to overlook the discontinuity in energy change with respect to the number of electrons and differentiate energy expressions directly with respect to the number of electrons. Thus one could differentiate (49) with respect to $N$ directly. This, however, requires one to ex- tend the definition of the electron propagator to noninteger numbers of electrons; it is not entirely clear how to do this.

\subsection{Approximations}

Chemical potential and hardness are usually calculated using finite difference approximations through (9) and (17) respectively. We have already shown that solving the Dyson equations provides accurate ionization potentials and electron affinities; thus, expressions for global reactivity indicators can be directly written in terms of the smallest ionization potential and the greatest electron affinity,

$$
\mu \approx-\frac{1}{2}(\underbrace{\min }_{k}\left(I_{k}\right)+\underbrace{\max _{l}}_{l}\left(A_{l}\right))
$$

and

$$
\begin{aligned}
\eta & \approx \underbrace{\min }_{k}\left(I_{k}\right)-\underbrace{\max }_{l}\left(A_{l}\right) \\
& \approx \underbrace{\min }_{k, l}\left(I_{k}-A_{l}\right) .
\end{aligned}
$$

Furthermore, within DFT formalism, there are other global reactivity descriptors defined in terms of $\mu$ and $\eta$ (whence depending on ionization potentials and electron affinities), that can be also determined using EPT values. For instance, the electrophilicity power of a chemical system reads, ${ }^{42}$

$$
\omega=\frac{\mu^{2}}{2 \eta} \approx \frac{(\underbrace{\min }_{k}\left(I_{k}\right)+\underbrace{\max _{l}}_{l}\left(A_{l}\right))^{2}}{8 \underbrace{\min }_{k, l}\left(I_{k}-A_{l}\right)} .
$$

Recently introduced concepts related to the quality of leaving groups can also be expressed in terms of ionization potentials and electron affinities, ${ }^{44,45}$

$$
\Delta E_{\text {nucleofuge }}=\frac{(\mu+\eta)^{2}}{2 \eta} \approx \frac{(\underbrace{\min _{k}}_{k}\left(I_{k}\right)-3 \underbrace{\max _{l}}_{l}\left(A_{l}\right))^{2}}{8 \underbrace{\min }_{k, l}\left(I_{k}-A_{l}\right)},
$$




$$
\Delta E_{\text {electrofuge }}=\frac{(\mu-\eta)^{2}}{2 \eta} \approx \frac{(3 \underbrace{\min }_{r}\left(I_{k}\right)-\underbrace{\min }_{l}\left(A_{l}\right))^{2}}{8 \underbrace{\min }_{k, l}\left(I_{k}-A_{l}\right)} .
$$

One advantage of using ionization potentials and electron affinities from electron propagator calculations is that, since the eigenvalues of (45) include correlation and orbital relaxation, the resulting reactivity indicators will also account for those effects. Moreover, there is a practical advantage since the ionization potential and electron affinity of a molecule are obtained from a single calculation of the electron propagator, while the conventional approach requires distinct energy calculations for the $N$, $N+1$, and $N-1$ electron systems.

\section{Local indicators}

\subsection{Exact theory}

Global indicators provide information about a global change in the chemical system under perturbation, but determining the specific site of the reactivity requires a local reactivity indicator. The electrostatic potential and the Fukui function play key roles in describing local reactivity. (Another key quantity is the polarizability kernel ${ }^{3,30,63}$

$$
P\left(\boldsymbol{r}, \boldsymbol{r}^{\prime}\right) \equiv\left(\frac{\delta^{2} E[v ; N]}{\delta v(\boldsymbol{r}) \delta v\left(\boldsymbol{r}^{\prime}\right)}\right)_{N}=\left(\frac{\delta \rho(\boldsymbol{r})}{\delta v\left(\boldsymbol{r}^{\prime}\right)}\right)_{N} .
$$

This can be evaluated using electron propagator theory, but the most direct approach employs the socalled polarization propagator. ${ }^{64}$ The polarizability kernel is intimately related to the softness kernel, and the two quantities are equal at zero temperature. ${ }^{63,65}$ ) The electrostatic potential is readily evaluated from the definition (18) and the equation for the electron density in terms of the Dyson orbitals (47). Computing the Fukui function from the results of an electron propagator calculation is more involved, insofar as the obvious approach (direct differentiation of the EPT expression for the electron density) does not immediately lead to a useful answer. Instead, we compute the Fukui function using

$$
f^{-}(\boldsymbol{r})=\left(\frac{\delta \mu^{-}}{\delta v(\boldsymbol{r})}\right)_{N}=\left(\frac{\delta(-I)}{\delta v(\boldsymbol{r})}\right)_{N},
$$

$$
f^{+}(\boldsymbol{r})=\left(\frac{\delta \mu^{+}}{\delta v(\boldsymbol{r})}\right)_{N}=\left(\frac{\delta(-A)}{\delta v(\boldsymbol{r})}\right)_{N}
$$

Importantly, this approach avoids the need to compute derivatives with respect to the number of electrons.

Using (45) and the definition of the pole strength, (48), we obtain an expression for the electronic chemical potential that is analogous to the common expression for the Hartree-Fock orbital energies,

$$
\begin{aligned}
& \mu^{-}[v] \equiv-I_{0}[v]= \\
& \frac{1}{p_{0}}\left(\int\left(\phi_{0}^{I P}(z)\right)^{*}\left(-\frac{\nabla^{2}}{2}+v(z)+j(z)-k(z)+\hat{\Sigma}\left(z,-I_{0}\right)\right)\right. \\
& \left.\phi_{0}^{I P}(z) \mathrm{d} z\right) .
\end{aligned}
$$

Here we have elected to denote the Dyson orbital and electron removal energy corresponding to the lowest ionization energy with a subscript zero.

Taking the functional derivative of both sides of (58) with respect to the external potential gives

$$
\begin{aligned}
& f^{-}\left(z^{\prime}\right)=\frac{1}{p_{0}} \int\left(\phi_{0}^{I P}(z)\right)^{*} \delta\left(z-z^{\prime}\right) \phi_{0}^{I P}(z) \mathrm{d} z \\
& +\frac{1}{p_{0}}\left\langle\phi_{0}^{I P}\left|\frac{\delta(j(z)-\hat{k}(z)+\hat{\Sigma}(z,-I))}{\delta v\left(z^{\prime}\right)}\right| \phi_{0}^{I P}\right\rangle \\
& +\frac{1}{p_{0}}\left(\left\langle\phi_{0}^{I P} \mid\left(\begin{array}{l}
-\frac{1}{2} \nabla^{2}+v(z)+j(z) \\
-\hat{k}(z)+\hat{\Sigma}(z,-I)
\end{array}\right) \frac{\delta \phi_{0}^{I P}(z)}{\delta v\left(z^{\prime}\right)}\right\rangle+c . c .\right. \\
& +\frac{-1}{p_{0}^{2}}\left(\frac{\delta p_{0}}{\delta v\left(z^{\prime}\right)}\right)\left\langle\begin{array}{l}
\phi_{0}^{I P}\left|\left(\begin{array}{l}
-\frac{1}{2} \nabla^{2}+v(z)+j(z) \\
-\hat{k}(z)+\hat{\Sigma}(z,-I)
\end{array}\right) \phi_{0}^{I P}\right\rangle \cdot
\end{array}\right.
\end{aligned}
$$

From (45) and the definition of the pole strength, (48), we conclude that the last two terms in (59) cancel out:

$$
-I_{0} \frac{\delta\left[p_{k}^{-1}\left\langle\phi_{0}^{I P} \mid \phi_{0}^{I P}\right\rangle\right]}{\delta v\left(z^{\prime}\right)}=-I_{0} \frac{\delta(1)}{\delta v\left(z^{\prime}\right)}=0
$$

Then, introducing the Hartree-Fock orbitals and using the chain rule for functional derivatives, the expression for the Fukui function can be simplified to 


$$
\begin{aligned}
& f^{-}\left(\mathbf{z}^{\prime}\right)= \frac{\left|\phi_{0}^{I P}\left(z^{\prime}\right)\right|^{2}}{p_{0}} \\
&+\frac{1}{p_{0}}\left(\iint\right.\left\{\left(\phi_{0}^{I P}(z)\right)^{*}\right. \\
& \times\left[\sum_{s} \frac{\delta(j(z)-k(z)+\hat{\Sigma}(z,-I))}{\delta \psi_{s}\left(z^{\prime \prime}\right)}\left(\frac{\delta \psi_{s}\left(z^{\prime \prime}\right)}{\delta v\left(z^{\prime}\right)}\right)_{N}\right] \\
&\left.\times \phi_{0}^{I P}(z) \mathrm{d} z^{\prime \prime} \mathrm{d} z\right\} \\
&\left.+\int\left(\phi_{0}^{I P}(z)\right)^{*}\left[\frac{\partial \hat{\Sigma}(z,-I)}{\partial I}\left(\frac{\delta I}{\delta v\left(z^{\prime}\right)}\right)_{N}\right] \phi_{0}^{I P}(z) \mathrm{d} z\right) .(61)
\end{aligned}
$$

Here, the index $s$ runs over all the Hartree-Fock orbitals, $\left\{\psi_{s}(z)\right\}$. The functional derivative of the Hartree-Fock orbitals with respect to the external potential can be computed using coupled-perturbed Hartree-Fock equations; relevant formulae are presented in the appendix. From (61), it is clear that

$$
f^{-}(\boldsymbol{r})=\left(p_{0}^{I P}\right)^{-1}\left|\phi_{0}^{I P}(\boldsymbol{r})\right|^{2}+\text { corrections, }
$$

and, by a similar argument to the preceding,

$$
f^{+}(\boldsymbol{r})=\left(p_{0}^{E A}\right)^{-1}\left|\phi_{0}^{E A}(\boldsymbol{r})\right|^{2}+\text { corrections. }
$$

In the Hartree-Fock model (where the self-energy is defined to be zero), the corrections are related to orbital relaxation. While this sort of orbital-based language is not entirely appropriate in this case, it is clear that the corrections are related to the relaxation of the orbitals (both the Hartree-Fock and the Dyson orbitals) that accompanies ionization or electron attachment. In particular, these formulae for the Fukui function take the form of a normalized Dyson orbital, plus suitable corrections.

To further simplify our expression for the Fukui function, we employ the expression for the pole strength in terms of the self-energy, (48), giving

$$
\begin{aligned}
& f^{-}\left(z^{\prime}\right)=\frac{\left|\phi_{0}^{I P}\left(z^{\prime}\right)\right|^{2}}{p_{0}} \\
&+\frac{1}{p_{0}}\left[\begin{array}{l}
\iint\left(\phi_{0}^{I P}(z)\right)^{*} \\
\times\left[\sum_{s} \frac{\delta(j(z)-k(z)+\hat{\Sigma}(z,-I))}{\delta \psi_{i}\left(z^{\prime \prime}\right)}\left(\frac{\delta \psi_{i}\left(z^{\prime \prime}\right)}{\delta v\left(z^{\prime}\right)}\right)_{N}\right] \\
\quad \times \phi_{0}^{I P}(z) \mathrm{d} z^{\prime \prime} \mathrm{d} z \\
+f^{-}(z)\left(p_{0}-1\right)
\end{array}\right]
\end{aligned}
$$

and so

$$
\begin{array}{r}
f^{-}\left(z^{\prime}\right)=\left|\phi_{0}^{I P}\left(z^{\prime}\right)\right|^{2} \\
+\iint\left(\phi_{0}^{I P}(z)\right)^{*}\left[\sum_{s} \frac{\delta(j(z)-k(z))}{\delta \psi_{s}\left(z^{\prime \prime}\right)}\left(\frac{\delta \psi_{s}\left(z^{\prime \prime}\right)}{\delta v\left(z^{\prime}\right)}\right)_{N}\right] \\
\times \phi_{0}^{I P}(\mathbf{z}) \mathrm{d} z^{\prime \prime} \mathrm{d} z \\
+\iint\left(\phi_{0}^{I P}(z)\right)^{*}\left[\sum_{s} \frac{\delta(\hat{\Sigma}(z,-I))}{\delta \psi_{s}\left(z^{\prime \prime}\right)}\left(\frac{\delta \psi_{s}\left(z^{\prime \prime}\right)}{\delta v\left(z^{\prime}\right)}\right)_{N}\right] \\
\times \phi_{0}^{I P}(z) \mathrm{d} z^{\prime \prime} \mathrm{d} z .
\end{array}
$$

This is the working equation for the nucleophilic Fukui function and, though the present derivation is different, the final result is formally identical to the expression of Cioslowski and Ortiz. ${ }^{66}$ (Note, however, that they use normalized Dyson orbitals in their derivation, which introduces an extra factor of the pole strength.)

A very similar derivation to the above gives an expression for the electrophilic Fukui function, namely,

$$
\begin{array}{r}
f^{+}\left(z^{\prime}\right)=\left|\phi_{0}^{E A}\left(z^{\prime}\right)\right|^{2} \\
\left.+\iint_{0}^{E A}(z)\right)^{*}\left[\sum_{s} \frac{\delta(j(z)-k(z))}{\delta \psi_{s}\left(z^{\prime \prime}\right)}\left(\frac{\delta \psi_{s}\left(z^{\prime \prime}\right)}{\delta v\left(z^{\prime}\right)}\right)_{N}\right] \\
\times \phi_{0}^{E A}(z) \mathrm{d} z^{\prime \prime} \mathrm{d} z \\
+\iint\left(\phi_{0}^{E A}(z)\right)^{*}\left[\sum_{s} \frac{\delta(\hat{\Sigma}(z,-I))}{\delta \psi_{s}\left(z^{\prime \prime}\right)}\left(\frac{\delta \psi_{s}\left(z^{\prime \prime \prime}\right)}{\delta v\left(z^{\prime}\right)}\right)_{N}\right] \\
\times \phi_{0}^{E A}(z) \mathrm{d} z^{\prime \prime} \mathrm{d} z
\end{array}
$$

where it is understood that the subscript zero indicates that we are considering the greatest electron affinity in the system (that is, the electron affinity that corresponds to the ground state of the anion).

In both (65) and (66), the Dyson Orbital is approximated as the square magnitude of the Fukui function, plus an "orbital relaxation" term that arises from Hartree-Fock theory, plus a final self-energy dependent term that corrects for all the effects of electron correlation (including the way electron correlation influences orbital relaxation). Note that because the pole strength is usually less than one, neglecting the last two terms in (65) and (66) would actually give a Fukui function that is not correctly normalized. For this reason, the "frozen orbital approximation" is not likely to be useful in electron propagator treatments of the Fukui function. 


\subsection{Approximations}

In order to derive approximate formulae for the Fukui functions, one need merely insert a specific approximation for the self energy (e.g., (46)) into (65) and (66). For practical work, it is most helpful to derive these corrections using an effective density matrix, whence the change in density associated with the $K$ th eigenstate of the propagator can be associated with

$$
\Delta \rho^{K}(z)=\sum_{p q} \Gamma_{p q}^{K} \psi_{p}^{*}(z) \psi_{q}(z)
$$

It follows from the derivation in the previous section that the first term in this expansion is just the Dyson orbital, ergo

$$
\Delta \rho^{K}(z)=\left|\phi_{K}^{(I P, E A)}\right|^{2}+\Delta \rho_{K}^{(\text {corr })}(z)
$$

Deriving the correction for any given choice of the self-energy is a tedious, but straightforward, exercise. For example, the second-order correction can be expressed as ${ }^{66,67}$

$$
\begin{aligned}
& \Delta \rho_{K}^{(2)}(z)=\sum_{p, q} d_{p K} d_{q K} \\
& {\left[\sum_{i j} \Gamma_{i j, p q}^{K}+\sum_{a b} \Gamma_{a b, p q}^{K}+\sum_{c k}\left(\Gamma_{k c, p q}^{K}+\Gamma_{c k, p q}^{* K}\right)\right]} \\
& \psi_{p}^{*}(z) \psi_{q}(z) .
\end{aligned}
$$

Here, $d_{p K}^{(I P, E A)}$ are expansion coefficients for the Dyson orbitals,

$$
\phi_{K}^{(I P, E A)}(\boldsymbol{z})=\sum_{p} d_{p K}^{(I P, E A)} \psi_{p}(\boldsymbol{z})
$$

The effective density matrix in (69) is broken into occupied-occupied,

$$
\begin{aligned}
\Gamma_{i j, p q}^{K}= & \sum_{c}\left[\sum_{k} \frac{\langle i k \| p c\rangle\langle q c \| j k\rangle}{\left(\varepsilon+\varepsilon_{c}-\varepsilon_{i}-\varepsilon_{k}\right)\left(\varepsilon+\varepsilon_{c}-\varepsilon_{j}-\varepsilon_{k}\right)}\right. \\
& \left.-\frac{1}{2} \sum_{d} \frac{\langle c d \| p j\rangle\langle q i \| c d\rangle}{\left(\varepsilon+\varepsilon_{i}-\varepsilon_{c}-\varepsilon_{d}\right)\left(\varepsilon+\varepsilon_{j}-\varepsilon_{c}-\varepsilon_{d}\right)}\right]
\end{aligned}
$$

virtual-virtual,

$$
\begin{aligned}
\Gamma_{a b, p q}^{K}= & \sum_{k}\left[\sum_{c} \frac{\langle a c \| p k\rangle\langle q k \| b c\rangle}{\left(\varepsilon+\varepsilon_{k}-\varepsilon_{a}-\varepsilon_{c}\right)\left(\varepsilon+\varepsilon_{k}-\varepsilon_{b}-\varepsilon_{c}\right)}\right. \\
& \left.-\frac{1}{2} \sum_{l} \frac{\langle k l \| p b\rangle\langle q a \| k l\rangle}{\left(\varepsilon+\varepsilon_{a}-\varepsilon_{k}-\varepsilon_{l}\right)\left(\varepsilon+\varepsilon_{b}-\varepsilon_{k}-\varepsilon_{l}\right)}\right]
\end{aligned}
$$

and off-diagonal (occupied-virtual)

$$
\begin{aligned}
& \Gamma_{k c, p q}^{K}=\Gamma_{c k}^{* K}=\left(\varepsilon_{k}-\varepsilon_{c}\right)^{-1} \\
& \left(\begin{array}{c}
\sum_{a}\left[\sum_{j} \frac{\langle c j \| p a\rangle\langle q a \| k j\rangle}{\left(\varepsilon+\varepsilon_{a}-\varepsilon_{c}-\varepsilon_{j}\right)}+\frac{1}{2} \sum_{b} \frac{\langle a b \| p k\rangle\langle q c \| a b\rangle}{\left(\varepsilon+\varepsilon_{c}-\varepsilon_{a}-\varepsilon_{b}\right)}\right] \\
-\sum_{i}\left[\sum_{b} \frac{\langle k b \| p i\rangle\langle q i \| c b\rangle}{\left(\varepsilon+\varepsilon_{i}-\varepsilon_{k}-\varepsilon_{b}\right)}+\frac{1}{2} \sum_{j} \frac{\langle i j \| p c\rangle\langle q k \| i j\rangle}{\left(\varepsilon+\varepsilon_{k}-\varepsilon_{i}-\varepsilon_{j}\right)}\right]
\end{array}\right)
\end{aligned}
$$

blocks. ${ }^{66,67}$ Higher order approximations have a similar form, but (71)-(73) are replaced by more complicated expressions.

There is a close link between the preceding analysis and previous work on computing adiabatic ionization potentials and electron affinities. Computing adiabatic ionization potentials and electron affinities requires information on the potential energy surface of the $N-1$ and $N+1$ electron systems, $V_{N-1}(\boldsymbol{R})$ and $V_{N+1}(\boldsymbol{R})$, in addition to the potential energy surface of the reference system $V_{0}(\boldsymbol{R})$. Letting $\lambda$ denote any parameter on which the external potential depends (of special interest are the nuclear coordinates), we have ${ }^{68}$

$$
\begin{aligned}
& \frac{\partial V_{N-1}(\boldsymbol{R})}{\partial \lambda}=\frac{\partial V_{0}(\boldsymbol{R})}{\partial \lambda}-\int f^{-}(\boldsymbol{x}) \frac{\partial v(\boldsymbol{x}, \boldsymbol{R})}{\partial \lambda} \mathrm{d} \boldsymbol{x}, \\
& \frac{\partial V_{N+1}(\boldsymbol{R})}{\partial \lambda}=\frac{\partial V_{0}(\boldsymbol{R})}{\partial \lambda}+\int f^{+}(\boldsymbol{x}) \frac{\partial v(\boldsymbol{x}, \boldsymbol{R})}{\partial \lambda} \mathrm{d} \boldsymbol{x} .
\end{aligned}
$$

These equations reduce to an especially simple form when they represent changes in geometry about the equilibrium geometry of the reference state, since then the first term on the right-hand-side of these equations is zero.

There have been a number of theoretical ${ }^{66,67}$ and computational $^{66,69-73}$ studies in which EPT approximations to (74) and (75) were used to compute adiabatic ionization potentials and electron affinities. It should not be difficult to modify those programs to extract the Fukui function. 


\section{Summary}

The preceding analysis elucidates the links between the density-functional theory (DFT) and electronpropagator theory (EPT) approaches to describing charge transfer. The EPT approach has much to recommend it, including the ability to systematically refine results towards the correct answer and arguably greater ability to represent the effects of electron correlation and orbital relaxation. The DFT approach, however, is more commonly used in qualitative and conceptual studies of chemical reactivity; the reactivity indicators associated with DFT are arguably simpler and easier to interpret than those associated with EPT. Here, by deriving accurate expressions for the conceptually useful DFT reactivity indices in terms quantities related to EPT, we have sought to combine the best points of both approaches. Not only is this approach more accurate, in some cases it may be more efficient, since three separate calculations using conventional quantum chemistry (one for the neutral, cation, and anion) are replaced by a single calculation using electron propagator theory.

Perhaps the most interesting results we obtain are (65) and (66), which show the relationship between the local quantities associated with electron removal and attachment in DFT (Fukui functions) and EPT (Dyson orbitals). Simply put, the Fukui function is equal to the square magnitude of the associated Dyson orbital, plus additional terms that can be related to orbital relaxation and electron correlation effects. Because such effects are very important when one forms an anion, we expect that the present approach will be useful for computing the Fukui function associated with electron attachment, $f^{+}(\boldsymbol{r})$. There is mounting evidence that the orbital relaxation that accompanies electron removal has an important,${ }^{49,50}$ and sometimes decisive, ${ }^{48}$ role in determining molecular reactivity preferences. Electron propagator approaches will be most important whenever orbital relaxation and/or electron correlation plays an important role in describing chemical reactivity. However, one cannot predict, a priori, when these effects may change molecular reactivity preferences. In this respect, pole strengths can be a useful indicator of the importance of these effects. In general, pole strengths between 0.8 and 1.0 indicate that the electron attachment and removal are well described by a simple Koopmans picture. Lower pole strengths indicate that higher order processes (such as ionizations coupled to excitations) are also important, which indicates that an accurate treatment of elec- tron correlation and orbital relaxation is essential. For this reason we hope that researchers wishing to make reliable reactivity predictions will consider using EPT, instead of Kohn-Sham methods, to compute reactivity indicators.

\section{Appendix A. Coupled-perturbed Hartree-Fock}

The functional derivative of the Hartree-Fock orbitals with respect to the external potential can be computed using the coupled-perturbed Hartree-Fock equations. These equations are commonly stated, however, for geometry changes, when the basis functions vary with respect to the external potential also. This is not the case of interest, here, which simplifies the analysis. The most useful form of the coupled-perturbed Hartree-Fock equations is that of Allen whose result, assuming real orbitals and adopting our notation, becomes ${ }^{74}$

$$
\begin{aligned}
& \left(-\frac{\nabla^{2}}{2}+v(z)+j(z)-k(z)\right)\left(\frac{\delta \psi_{i}(z)}{\delta v\left(z^{\prime}\right)}\right)_{N} \\
& =\left(-\delta\left(z-z^{\prime}\right)+\left(\frac{\delta \varepsilon_{i}}{\delta v\left(z^{\prime}\right)}\right)\right) \psi_{i}(z) \\
& -\sum_{s}\left(\int \frac{\psi_{s}\left(z^{\prime \prime}\right) \psi_{i}\left(z^{\prime \prime}\right)}{\left|\boldsymbol{r}-\boldsymbol{r}^{\prime \prime}\right|} \mathrm{d} z^{\prime \prime}\right)\left(\frac{\delta \psi_{s}(z)}{\delta v\left(z^{\prime}\right)}\right)_{N} \\
& -\sum_{s}\left(\int \frac{\left(\frac{\delta \psi_{s}\left(z^{\prime \prime}\right)}{\delta v\left(z^{\prime}\right)}\right)_{N} \psi_{i}\left(z^{\prime \prime}\right)}{\left|\boldsymbol{r}-\boldsymbol{r}^{\prime \prime}\right|} \mathrm{d} z^{\prime \prime}\right) \psi_{s}(\boldsymbol{z}) \\
& +2 \sum_{s}\left(\int \frac{\psi_{s}\left(\mathbf{z}^{\prime \prime}\right)\left(\frac{\delta \psi_{s}\left(z^{\prime \prime}\right)}{\delta v\left(z^{\prime}\right)}\right)_{N}}{\left|r-r^{\prime \prime}\right|} \mathrm{d} z^{\prime \prime}\right) \psi_{i}(z)
\end{aligned}
$$

where

$$
\begin{aligned}
\left(\frac{\delta \varepsilon_{i}}{\delta v\left(z^{\prime}\right)}\right)_{N}= & \left|\phi_{i}\left(\left(z^{\prime}\right)\right)\right|^{2} \\
& {\left[\begin{array}{c}
\left(\psi_{s}\left(z^{\prime \prime}\right) \frac{\delta \psi_{s}\left(z^{\prime \prime}\right)}{\delta v\left(z^{\prime}\right)}\right)\left|\psi_{i}(z)\right|^{2} \\
-2 \sum_{s} \iint \frac{\left[\psi_{s}\left(z^{\prime \prime}\right) \psi_{i}\left(z^{\prime \prime}\right)\right)\left(\psi_{i}(z) \frac{\delta \psi_{s}(z)}{\delta v\left(z^{\prime}\right)}\right)}{\left|\boldsymbol{r}-\boldsymbol{r}^{\prime \prime}\right|} \mathrm{d} z \mathrm{~d} z^{\prime \prime}
\end{array}\right.}
\end{aligned}
$$


Here, the index $s$ runs over all the Hartree-Fock orbitals (both occupied and virtual). Solving (A1) with respect to the orthogonality conditions on the orbitals gives the desired solution.

In practice, one introduces a basis set; if one expands the perturbed orbitals in terms of the unperturbed orbitals, with

$$
\frac{\delta \psi_{s}(z)}{\delta v\left(z^{\prime}\right)}=\sum_{t} u_{s t}\left(z^{\prime}\right) \psi_{t}(z)
$$

multiplies (A1) by $\psi_{l}(z)$, and integrates with respect to $z$, then one obtains the common form of the coupledperturbed Hartree-Fock equations. ${ }^{75,76}$ Inasmuch it is inconvenient for the coefficients of expansion in (A3) to depend on the point at which the perturbation occurs, it is sometimes helpful to then rewrite the equation as

$$
u_{s t}\left(z^{\prime}\right)=\sum_{q r} w_{q r s t} \psi_{q}^{*}\left(z^{\prime}\right) \psi_{r}\left(z^{\prime}\right)
$$

and then

$$
\frac{\delta \psi_{s}(z)}{\delta v\left(z^{\prime}\right)}=\sum_{q r t} w_{q r s t} \psi_{q}^{*}(z) \psi_{r}\left(z^{\prime}\right) \psi_{t}(z)
$$

Using this form, one can derive the corrections in terms of the transition density matrix, as in §5.2.

\section{Acknowledgements}

PWA would like to acknowledge support from NSERC and the Canada Research Chairs. The National Science Foundation provided support through a grant (CHE-0135823) to Kansas State University.

\section{References}

1. Ayers P W and Yang W 2003 Density functional theory (eds) P Bultinck, H de Winter, W Langenaeker and J P Tollenaere (New York: Dekker) pp 571-616

2. Kohn W, Becke A D and Parr R G 1996 J. Phys. Chem. 10012974

3. Parr R G and Yang W 1989 Density-functional theory of atoms and molecules (New York: Oxford University Press)

4. Dreizler R M and Gross E K U 1990 Density functional theory: An approach to the quantum manybody problem (Berlin: Springer-Verlag)
5. Kohn W 1999 Rev. Mod. Phys. 711253

6. Geerlings P, De Proft F and Langenaeker W 2003 Chem. Rev. 1031793

7. Kohn W and Sham L J Phys. Rev. 140 A1133-A1138

8. McWeeny R and Pickup B T 1980 Rep. Prog. Phys. 431065

9. Herrera B, Dolgounitcheva O, Zakrzewski V G, Toro-Labbe A and Ortiz J V 2004 J. Phys. Chem. A108 11703

10. Ortiz J V 1999 Adv. Quantum Chem. 3533

11. Ohrn Y and Born G 1981 Adv. Quantum Chem. 131

12. Yang W, Parr R G and Pucci R 1984 J. Chem. Phys. 812862

13. Parr R G and Yang W 1984 J. Am. Chem. Soc. 106 4049

14. Gritsenko $\mathrm{O} V$, Ensing $\mathrm{B}$, Schipper $\mathrm{P} R \mathrm{R}$ and Baerends E J 2000 J. Phys. Chem. A104 8558

15. Zhang Y and Yang W 1998 J. Chem. Phys. 1092604

16. De Proft F, Vivas-Reyes R, Peeters A, Van Alsenoy C and Geerlings P 2003 J. Comput. Chem. 24463

17. Ayers P W, Anderson J S M and Bartolotti L J 2004 Int. J. Quantum Chem. 101520

18. Hohenberg P and Kohn W 1964 Phys. Rev. 136 B864-B871

19. Yang W T, Ayers P W and Wu Q 2004 Phys. Rev. Lett. 92

20. Freed K F and Levy M 1983 J. Chem. Phys. 77396

21. Levy M 1979 Proc. Natl. Acad. Sci. USA 766062

22. Lieb E H 1983 Int. J. Quantum Chem. 24243

23. Ayers P W, Lucks J B and Parr R G 2002 Acta Chim. Phys. Debricina 34-35 223

24. Parr R G, Donnelly R A, Levy M and Palke W E 1978 J. Chem. Phys. 683801

25. Itzkowski R P and Margrave J L $1961 \mathrm{~J}$. Am. Chem. Soc. 833547

26. Mulliken R S 1934 J. Chem. Phys. 2782

27. Senet P 1996 J. Chem. Phys. 1056471

28. Senet P 1997 J. Chem. Phys. 1072516

29. Ayers P W and Parr R G 2000 J. Am. Chem. Soc. 122 2010

30. Ayers P W and Parr R G 2001 J. Am. Chem. Soc. 123 2007

31. Nalewajski R F 1983 J. Chem. Phys. 786112

32. Klopman G 1968 J. Am. Chem. Soc. 90223

33. Parr R G and Pearson R G 1983 J. Am. Chem. Soc. 1057512

34. Sanderson R T 1951 Science 114670

35. Pearson R G 1987 J. Chem. Educ. 64561

36. Pearson R G 1999 J. Chem. Educ. 76267

37. Parr R G and Chattaraj P K 1991 J. Am. Chem. Soc. 1131854

38. Chattaraj P K 1996 Proc. Indian Natl. Sci. Acad. $\mathbf{A 6 2}$ 513

39. Pearson R G 1963 J. Am. Chem. Soc. 853533

40. Chattaraj P K, Lee H and Parr R G 1991 J. Am. Chem. Soc. 1131855

41. Ayers P W 2005 J. Chem. Phys. 122141102

42. Parr R G, Von Szentpaly L and Liu S B 1999 J. Am. Chem. Soc. 1211922

43. Chattaraj P K and Maiti B 2001 J. Phys. Chem. A105 169 
44. Ayers P W, Anderson J S M and Bartolotti L J 2005 Int. J. Quantum Chem. 101520

45. Ayers $\mathrm{P}$ W, Anderson J S M, Rodriguez J I and Jawed Z 2005 Phys. Chem. Chem. Phys. 71918

46. Politzer P and Truhlar D 1981 Chemical applications of atomic and molecular electrostatic potentials (New York: Plenum)

47. Ayers P W and Levy M 2000 Theor. Chem. Acc. 103 353

48. Bartolotti L J and Ayers P W 2005 J. Phys. Chem. A109 1146

49. Flurchick K and Bartolotti L 1995 J. Mol. Graph. 13 10

50. Langenaeker W, Demel K and Geerlings P 1991 Theochem 80329

51. Perdew J P, Parr R G, Levy M and Balduz Jr J L 1982 Phys. Rev. Lett. 491691

52. Yang W, Zhang Y and Ayers P W 2000 Phys. Rev. Lett. 845172

53. Gritsenko O V, Braida B and Baerends E J $2003 \mathrm{~J}$. Chem. Phys. 1191937

54. Chong D P, Gritsenko O V and Baerends E J $2002 \mathrm{~J}$. Chem. Phys. 1161760

55. Savin A, Umrigar C J and Gonze X 1998 Chem. Phys. Lett. 288391

56. Levine Z H and Allan D C 1989 Phys. Rev. Lett. 63 1719
57. Godby R W, Schluter M and Sham L J Phys. Rev. B36 6497

58. Godby R W, Schluter M and Sham L J 1988 Phys. Rev. B37 10159

59. Sham L J and Schluter M 1983 Phys. Rev. Lett. 511888

60. Sham L J and Schluter M 1985 Phys. Rev. B32 3883

61. Duffy P, Chong D P, Casida M E and Salahub D R 1994 Phys. Rev. A50 4707

62. Stillinger F H 2000 J. Chem. Phys. 1129711

63. Berkowitz M and Parr R G 1988 J. Chem. Phys. 88 2554

64. McWeeny R 1989 Methods of molecular quantum mechanics 2nd edn (London: Academic Press)

65. Ayers P W 2001 Theor. Chem. Acc. 106271

66. Cioslowski J and Ortiz J V 1992 J. Chem. Phys. 968379

67. Ortiz J V 2000 J. Chem. Phys. 11256

68. Ayers P W and Parr R G 2000 J. Phys. Chem. A104 2211

69. Ortiz J V 1993 Chem. Phys. Lett. 216319

70. Ortiz J V 1993 Chem. Phys. Lett. 214467

71. Ortiz J V 1993 J. Chem. Phys. 996716

72. Ortiz J V 1993 J. Chem. Phys. 996727

73. Ortiz J V 1992 J. Chem. Phys. 977531

74. Allen L C 1960 Phys. Rev. 118167

75. Peng H W 1941 Proc. R. Soc. London A178 499

76. Stevens R M, Pitzer R M and Lipscomb W N 1963 J. Chem. Phys. 38550 